Series A

I. MATHEMATICA

329

\title{
ON THE NORM OF A LINEAR \\ OPERATOR IN A HILBERT SPACE
}

BY

TOIVO NIEMINEN

H E L S I N K I 1962

S U O M A L A I N E T I E D E K A T E M A

https://doi.org/10.5186/aasfm.1963.329 
Communicated 12 October 1962 by R. Nevanlinna and Olli Lehto 


\section{On the norm of a linear operator in a Hilbert space}

The purpose of this paper is to prove the following theorem:

Let $T$ be a linear mapping of a real or complex Hilbert space $\mathfrak{5}$ into

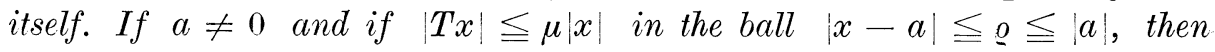
$|T x| \leqq \frac{\mu|a|}{\varrho}|x|$ for every $x$ in $\mathfrak{H}$.

We may restrict ourselves to the case when $\varrho<|a|$. In fact, if the theorem is valid for all $\varrho$ and $a$ with $\varrho<|a|$, and if $|T x| \leqq \varrho|x|$ for every $x$ satisfying $|x-a| \leqq|a|$, then $|T x| \leqq \frac{\mu|a|}{\varrho}|x|$ for every $\varrho<|a|$, and, consequently,

$$
|T x| \leqq \inf _{\varrho<|a|} \frac{\mu|a|}{\varrho}|x|=\frac{\mu|a|}{|a|}|x| .
$$

On the other hand, from the linearity it immediately follows, that we may suppose $|a|=1$.

Let $\Re$ be the tangent cone of the sphere $|x-a|=\varrho$ with the vertex 0 . From the linearity it follows, that $|T x| \leqq \mu|x|$ for every $x$ inside $\Re$. In other words, if $\lambda$ is such that $(\lambda x-a, x)=0$, i.e. $\lambda=\frac{(a, x)}{|x|^{2}}$, and if $|\lambda x-a| \leqq \varrho$, then $|T(\lambda x)| \leqq \mu|\lambda||x|$ and, consequently, $|T x|$ $\leqq \mu|x|$. The condition $|\lambda x-a| \leqq \varrho$ can be written

or

$$
\varrho^{2}|x|^{4}=\left.\left.|(a, x) x-| x\right|^{2} a\right|^{2}=|(a, x)|^{2}|x|^{2}+|x|^{4}-2|x|^{2} \mid(a, x \mid)^{2}
$$

$$
\left(1-\varrho^{2}\right)|x|^{2} \leqq|(a, x)|^{2}
$$

Thus $|T x| \leqq \mu|x|\left(\leqq \frac{\mu}{\varrho}|x|\right)$ for every $x$ satisfying (1).

Now we consider a vector $x$ lying outside of $\AA$. From the preceding it follows that if $x+\lambda a$ lies in $\Re$, i.e. if it satisfies the inequality

$$
\left(1-\varrho^{2}\right)|x+\lambda a|^{2} \leqq \mid(x+\lambda a, a)^{2},
$$


then

$$
|T(x+\lambda a)| \leqq \mu|x+\lambda a|
$$

or

(3) $|T x|^{2}+|\lambda|^{2}|T a|^{2}+2 \operatorname{Re}\{\lambda(T a, T x)\} \leqq|\mu|^{2}\left[|x|^{2}+\mid \lambda^{2}+2 \operatorname{Re}\left\{\lambda^{2}(a, x)\right\}\right]$.

The inequality (2) can be written

$$
\left(1-\varrho^{2}\right)\left(|x|^{2}+|\lambda|^{2}+2 \operatorname{Re}\{\lambda(a, x)\}\right) \leqq\left|(x, a)^{2}+\right| \lambda^{2}+2 \operatorname{Re}\{\lambda(a, x)\}
$$

or

$$
\left.\varrho^{2}\left|\lambda^{2}+2 \varrho^{2} \operatorname{Re}\{\lambda(a, x)\}+\right|(x, a)\right|^{2}-\left(1-\varrho^{2}\right)|x|^{2} \geqq 0 .
$$

We first consider the case when $\mathfrak{H}$ is a complex Hilbert space. If $\lambda$ satisfies the conditions

$$
\operatorname{Re}\{\lambda(T a, T x)\}=0
$$

and

$$
\left.\varrho^{2} \lambda_{i}^{2}+2 \varrho^{2} \operatorname{Re}\{\lambda(a, x)\}+(x, a)\right\}^{2}-\left(1-\varrho^{2}\right) x^{2}=0,
$$

then

$$
x^{2}+|\lambda|^{2}+2 \operatorname{Re}\{\lambda(a, x)\}=\frac{x^{2}-(x, a)^{2}}{\varrho^{2}} \leqq \frac{x^{2}}{\varrho^{2}} .
$$

and, by virtue of (3),

(6) $\left|T x^{2} \leqq T x\right|^{2}+|\lambda|^{2} \mid T a^{2} \leqq \mu^{2}\left(x^{2}+\lambda^{2}+2 \operatorname{Re}\{\lambda(a, x)\}\right) \leqq \frac{\mu^{2} x^{2}}{\varrho^{2}}$.

We thus see, that the theorem holds in the complex case if it is possible to choose $\lambda$ in such a way that it satisfies both equations (4) and (5).

Since $x$ lies outside of $\mathfrak{K}$, it satisfies the condition

$$
\left.\left|(a, x)^{2}<\left(1-\varrho^{2}\right)\right| x\right|^{2} .
$$

From this it follows that for the real function

$$
f(\lambda)=\left.\varrho^{2}\left|\lambda^{2}+2 \varrho^{2} \operatorname{Re}\{\lambda(a, x)\}+\right|(x, a)\right|^{2}-\left(1-\varrho^{2}\right) \mid x^{2}
$$

there is

$$
f(0)=|(x, a)|^{2}-\left(1-\varrho^{2}\right):\left.x\right|^{2}<0 .
$$

Since, furthermore, $\lim _{\lambda \rightarrow \infty} f(\lambda)=\infty$ and since $f$ is continuous, the equation $f(\lambda)=0$ thus has a solution on every straight line through the origin. On the other hand, we immediately see, that there is a straight line through 
the origin on which $\operatorname{Re}\{\lambda(T a, T x)\}$ vanishes identically. Thus the equations (4) and (5) always have a common solution $\lambda$, and our theorem is valid in the complex case.

If $\mathfrak{S g}$ is real and if, for example, $(a, x) \geqq 0$, then

$$
\operatorname{Re}\{\lambda(a, x)\}=\lambda(a, x) \geqq 0
$$

for all $\lambda \geqq 0$. Exactly in the same way as above we see, that the equation (5) has a solution $\lambda>0$. For this $\lambda$ we then get the inequality (6) as before, and our theorem is valid also in the real case.

It is readily seen, that the evaluation given above cannot be improved. It suffices to take the 2-dimensional Hilbert space $\mathfrak{S}$ with the basis vectors $a_{1}$ and $a_{2}$, and consider the linear operator $T$ defined by the equations $T a_{1}=0$ and $T a_{2}=\alpha a_{2}$ with $|\alpha|>1$. For the vector

we then get

$$
x=e^{i \varphi}|x|\left(a_{1} \cos \psi+a_{2} \sin \psi\right)
$$

and, consequently,

$$
T x=e^{i \varphi} x: \alpha \sin \psi
$$

$$
|T x| \leqq \alpha \rho \mid
$$

in the cone $\sin \psi \leqq \varrho<1$, and $\mu=\alpha \varrho$ is the smallest real number satisfying this inequality. For the operator $T$ we thus have

$$
\mid T_{i}=\alpha_{1}=\frac{\mu}{\varrho} .
$$

